\title{
MULTIPLE WORLDS IN SIMULATION GAMES FOR SPATIAL DECISION MAKING: CONCEPT AND ARCHITECTURE
}

\author{
Michele Fumarola \\ Alexander Verbraeck \\ Systems Engineering Group \\ Faculty of Technology, Policy and Management \\ Delft University of Technology \\ Jaffalaan, 5, 2628BX, Delft, THE NETHERLANDS
}

\begin{abstract}
In this paper we present the use of "what-if"-analysis in simulation games for spatial decision making by introducing the concept of multiple worlds. We expect that "what-if"analysis in games enables the trainees to achieve more robust results, which is defined as the ability to achieve the required goals given different scenarios. Scenarios are defined as exogenous variables on the multiple worlds. Viable decisions for a particular world are assessed by splitting the given world into multiple ones and running simulations for these new worlds. This assessment is performed by a) comparing the alternatives represented by each world and b) exploring the timeline of each world by selecting specific time instants. The navigation both through multiple worlds and through time should provide the users the possibility to formulate a robust answer for the specified problem. We will present a viable architecture and 4 distinct modes of game-play for the simulation game.
\end{abstract}

\section{INTRODUCTION}

Spatial decision making generally involves ill structured problems with a spatial component in which multiple actors are involved. There is a large variety of problems requiring spatial decision making e.g. the development of airports and seaports. The best known examples of spatial decision making processes are found in large construction projects. These projects are often plagued by underestimation of costs and time and by other unexpected problems. Various examples are found in literature e.g. the Denver International Airport lasting 2 years longer and costing \$3 billions more than expected (Szyliowicz and Goetz 1995) and transport infrastructure projects like bridges and tunnels costing on average $33.8 \%$ more than expected (Flyvbjerg, Holm, and Buhl 2004). Simulation has often been suggested as an inquiry system for ill structured problems. Simulation games, as described by Galvao, Martins, and Gomes (2000), are a specific kind of simulations which can be used for educational and training purposes, especially in cases where the interaction between participants plays a role. In this paper, we want to present a specific simulation game for spatial decision making where the concept of multiple worlds is used to provide insight into the problem. "Multiple worlds" denotes the concept of having a number of the same set of objects for which decisions have to be made. A set of objects forms a unique world. This concept allows users to compare alternatives by assessing different decisions on multiple copies of the same world, which each having a slightly different state. This comparison is shown to be useful in decision making in order to achieve robust results (Hybinette and Fujimoto 2001). The multiple worlds concept is in that sense very close to the "what-if" nature of simulation modeling in general. In most simulation studies we create several versions of the same model for experimentation with different parameter sets (Goldsman and Nelson 1998). The simulation game we propose consists of different components: a multi-actor environment, a game, multiple worlds, visualization and simulation. We propose distinct tools for and modes of game-play to use multiple worlds in simulation games. Furthermore we introduce an architecture able to couple the different components in order to assess the feasibility and usability of the presented simulation game.

\section{BACKGROUND}

\subsection{Supporting spatial decision making}

Support for spatial decision making processes can be discussed more easily if framed in a specific context. Ports and industrial sites are just a couple of examples in which spatial decision making plays a crucial role during the designing phases of such areas. (Chin 2007) performed extensive research in the area of mainport planning and the way in which a studio-based approach could benefit the process. During the initial observations during the case study conducted at the Port of Rotterdam, little use of technology 


\section{Fumarola and Verbraeck}

during meetings as the information was mainly paper-based. This introduction of software services in a studio-based approach provided a solution wherein the use of technology can improve the effectiveness of the actors involved in spatial decision making. This shift from paper-based information to software services in a studio-based approach has been pushed forward towards networked virtual environments, although mainly in gaming environments (e.g. Guy, Bidwell, and Musumeci (2005)). Whereas actors previously needed to be present on the same location at the same time in order to make decisions, the use of technology is now able to remove this barrier as well.

\subsection{D Virtual environments for collaboration and visualization}

Numerous amount of research has been done applicable to 3D virtual environments (3DVE) to support spatial decision making: e.g. interaction in 3DVEs, 3DVEs as educational environments (Educational Virtual Environments), as a support for collaboration (Collaborative Virtual Environments) and as tools for visualization e.g. Bouras et al. (2006), Pekkola (2002). As these 3D virtual environments can also be connected in networked virtual environments, collaboration becomes possible with remote users. This makes this kind of environments more interesting for projects where people have to collaborate from different places (e.g. companies with offices located in different places worldwide). As these virtual environments are also used to visualize information (e.g. representation of the physical world on which users have to make decisions), users can have an integrated set of tools wherein collaboration, visualization and communication is fully supported.

\subsection{Simulation games for spatial decision making}

Simulation games have often been found useful as a method of inquiry to gain insight in complex system behavior and as aids for training and education. Various examples exist of simulation games for spatial decision making to gain insight into the decision making process. The main focus of these simulation games is to provide a safe environment to assess decisions and their consequences. SimPort-MV2 (Bekebrede and Mayer 2006) is an example of such a game: the goal of the game is to design and allocate land for the Maasvlakte-2 port area in the Port of Rotterdam. Players gain insight into the development strategies, the multidisciplinary thinking accompanied by such a project and the negotiation process. For the SimPort-MV2 game, the decision process itself has also been studied, and tools to support the decision makers in making complex trade-offs for the development of the port have been studied as well (Chin 2007). The study of the real decision making process made it clear that many alternatives are compared in reality, and that the decision makers spend a lot of time looking at the consequences of choosing a certain alternative. However the simulation games often fail to provide sufficient support to compare alternatives in order to find the most fulfilling solution to the presented situation, requirements and goals. In most simulation games, time is represented as the linear flow of events as perceived in reality and there is only a single instance of the simulation representing the real world to interact with. The possibility to revisit earlier made decisions enriches the learning experience provided by a simulation game, as it already does in a normal simulation study through its "what-if" nature.

\subsection{Multiple worlds to compare alternatives}

Although used often in literature and natural sciences (Vaidman 1998), the concept of multiple worlds (depending on the field of study also referenced as alternative reality, multiverse, many-worlds, etcetera) has seldom been used in simulation and simulation games. Rare examples include (Franks et al. 1997) and Hybinette and Fujimoto (2001). The work of Franks (Franks et al. 1997) describes time warps as a functionality to assess different alternatives by subsequently performing rollbacks in time for each alternative. The work of Hybinette (Hybinette and Fujimoto (1997), Hybinette and Fujimoto (2001), Hybinette (2004)) proposes an architecture to clone worlds allowing the users of the simulation to explore and compare alternatives that run simultaneously. Except for some entertainment games with multiple worlds as a minor feature (i.e. to explain illogical events in the storyline), specific instances of simulation games presenting multiple worlds as a functionality to support the exploration of alternative decisions are found to be not at hand. This gap in the literature also suggests that implementations of multiple worlds in simulation games for an educational setting nonexistent. This paper provides a first architecture for such a simulation game.

\section{MULTIPLE WORLDS AS A TEACHING CONCEPT IN SPATIAL DECISION MAKING}

\subsection{Overview of the use of multiple worlds for spatial decision making}

The ability to compare alternatives before taking a decision allows actors in a spatial decision making process to make more informed decisions. Where in standard games users only can influence on a limited number of variables for which they can change the values, spatial decision making involves changes that have a much broader impact. Often, decision makers want to research completely different solutions from the one first thought of. If we consider the example of a spatial decision making process for the development of an airport, the actors involved should be 


\section{Fumarola and Verbraeck}

able to freely explore multiple possibilities (e.g. location of runways, requirements to accommodate certain numbers of passengers) within specific constraints (e.g. budget and time). These decisions will take place at a specific point in time in the game, where the simulation model in the game has a specific state, which provides the opportunity to explore the alternative. Choosing to implement this decision or not leads to two (or more) branches of virtual reality that can be further explored during game-play. When multiple decisions like this are taken in the game, a tree can be constructed with a node for each decision. Users should be able to come back to these decisions at a later stage to explore the other branch. In addition, the simulation model itself will have stochastic effects that may or may not take place, which will have an influence on model outcomes. At any time during the decision making process, the possibility will be given to use scenarios in order to assess the robustness of the result by looking at one of the scenarios based on the decision trees, and exposing it to stochastic events in a number of "replications" of the model with varying conditions. In case the actors are not satisfied with the result, they will be able to change the made decisions by re-assessing them in the constructed tree. By having the possibility of just reconsidering decisions that lead to a less than optimal result, they do not need to redo the whole decision making process but only the stages that are considered problematic.

\subsection{Requirements of tools needed}

Neither the current gaming tools, nor the standard simulation tools or virtual reality tools are able to support the multiple worlds concept for training simulations. Instead, we need a well-integrated set of tools. These tools should make it possible to manipulate and navigate the worlds in an appropriate way as to allow the users to gain understanding of the structure of the solution space and the solution contents.

We recognize the following tools as essential in this context:

- Branch: branching of the solution space should be possible at arbitrary moments, after a set of decisions, and automatically at certain points in time. The latter should also be possible by attaching a condition to which the branching takes place. Users should be able to insert new branches at a later stage, for instance when they see that a decision that was made leads to an unwanted or non-robust outcome.

- Merge: given two (or more) states, we should be able to merge these into one state and still be able to extract the decisions leading to this new merged state. This is especially important if multiple spatial decisions have been made, and the users want to research the combined effect of the decisions.

- Rollback and prune: after a user entered a number of decision, it is often the case this sequence of decisions does not lead to an acceptable state, or the user believes a better outcome is still attainable. Another possible case would be the one in which a user continues with a faulty state and introduces multiple decisions and solutions but afterwards decides on going back to a previous state. In both cases the user would prefer to delete all states that do not lead to good solutions thus rolling back to a previous state and pruning the subtree that is not necessary anymore.

- Navigate: a user should easily go from one state to another by following the structure of the tree as to clearly comprehend the changes that take place after a specific decision or solution is introduced into the current state.

- Compare: the user should be able to take two arbitrary state and manually compare those by visualizing both states in a consistent way. Consistency is achieved here by the concurrent visualization of a comparable subset of the states. In addition to visualization, statistical comparisons between different states can also be provided, either for different branches of the decision tree at the same point in time (e.g., to compare end-states), or for different times in the same branch (e.g., to study progress).

\subsection{Gameplay modes}

Introducing multiple worlds in a simulated gaming environment is certainly a challenging task. Giving full control to the player over the multiple worlds can provide a fulfilling experience in terms of achieving different learning goals. However we can also restrict this control to a small subset of possibilities for which users would learn to achieve their goals. This would limit the need for extremely complex and fast tools, while still being able to achieve the teaching goals.

Starting from the previous reasoning, we can distinguish a number of possible gaming modes for which different game-play behaviors apply. The game-play behaviors would go from full control of manipulation and navigation of the multiple worlds to limited control where user-induced branching, merging, or rollback/pruning would not be possible. This would lead us to the following possibilities:

- Full control: the players can navigate and manipulate (branch, merge, rollback, prune) the multiple worlds without any restrictions and only have to take into consideration the final goal of the game. 
This final goal can be a growth percentage they have to achieve, keeping the total costs of the project to a minimum, etc.

- Rollback and branch: the players can only do rollbacks in the multiple world space and make a branch after a rollback one-at-a-time. Merging, pruning, and comparing multiple alternatives in parallel are not possible. This would restrict the possibility and complexity for the users, but still allow the players to redo their decisions and solutions in case of mistakes.

- Set of decisions: the players are not allowed to define the decisions they want to introduce, but have to choose from a set of decisions and branches given by the game scenario. It is important to introduce them at the right moment during the gaming timeline. Depending on the teaching goal, one could allow the players to explore alternative branches later or not.

- From start to end: the start and end state are given for which the players should define the intermediate path. This intermediate path should lead to the end state or an end state that is close enough to the given end state. This can be checked based on a set of thresholds for different state variables. The unique aspect is that the multiple world concept provides a way to show the desired end state to the users, and that the users can study the end-state at length before defining their path from the start state.

\section{POSSIBLE SCENARIOS}

Chin (2007) and Bekebrede and Mayer (2006) provide some interesting case studies to elaborate upon. We will present scenarios based on these cases to share our contribution by pointing out the differences with existing solutions. We will therefore present 4 different scenarios: spatial decision making supported by paper-based information, spatial decision making with a studio-based approach, support for spatial decision making with simulation games for training and finally with multiple worlds in simulation games for training. Figure 1 (pictures provided by Chin (2007) and $<$ wWW. simport. eu>) depicts the progress in a diagram to show the evolution from paper-based approach to the concept presented in this paper.

\subsection{Paper-based information}

A group of professionals (e.g. engineers and financial advisors) are in charge of exploring different possibilities on the extension of an existing harbor area. To work on this task, they decide to organize workshops wherein they study existing documentation and reports in order to generate a number of viable possibilities. These workshops are rather unstructured as they do not have support to collect information in an orderly fashion and to guide discussions by having clear overviews of the different ideas that come up during the workshops. As the case they work on, turns up to be rather unique (they therefore cannot use existing projects as reference material) and tedious, they decide to work on a single design which they will use to base a simulation study upon. Different consultations with simulation experts over an extended time period, help them understand and resolve aspects of the design which had a negative influence on the result they initially hoped for. This process of trialand-error proceeds until a design is found that generates the wished results.

\subsection{Studio-based approach}

A group of professionals (e.g. engineers and financial advisors) are in charge of exploring different possibilities on the extension of an existing harbor area. The workshops they organize are supported by IT services existing out of web services available online. The participants of the workshop enter their ideas in the IT tools to their disposal, as such they always have a clear overview of the discussions made and existing information. As the case they work on, turns up to be rather unique (they therefore cannot use existing projects as reference material) and tedious, they decide to work on a single design which they will use to base a simulation study upon. As the information they collected during the workshops and brainstorming sessions is well structured, consultations with simulation experts go smoothly and communication goes quicker. Although the results from the simulations are rather disappointing, the subsequent discussions about the initial design lead to better results as the simulation study can be visualized during the meetings.

\subsection{Simulation games for training}

A group of professionals (e.g. engineers and financial advisors) are in charge of exploring different possibilities on the extension of an existing harbor area. As the case they work on, turns up to be rather unique (they therefore cannot use existing projects as reference material) and tedious, they decide to use a simulation game in order to gain better insight into this complex problem. During the game, a simulation is in charge of displaying what could happen after certain decisions. As the game offers a safe environment to work in, the professionals feel more comfortable to explore more risky decisions. Although the game offers a good support to freely explore the problem, the professionals need different gaming sessions to assess different possibilities, as they need to start from scratch for every possibility. 


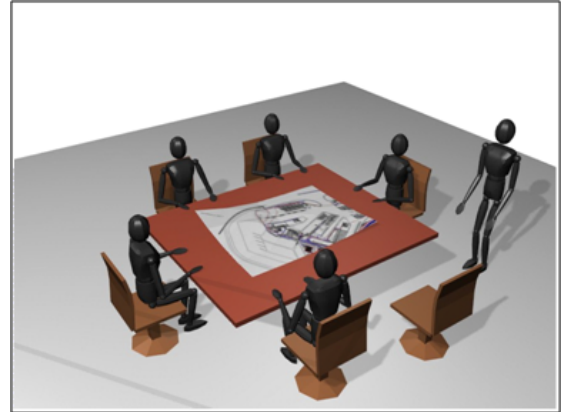

(1)

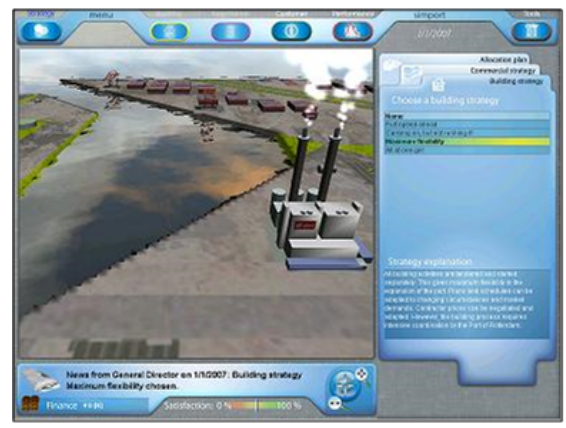

(3)
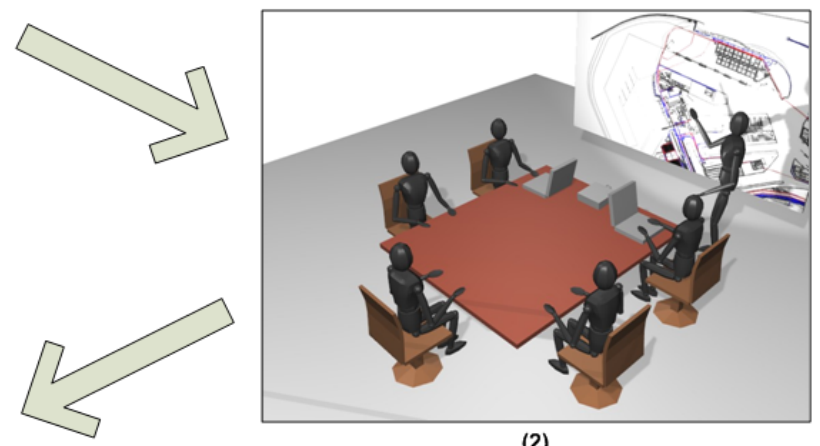

(2)
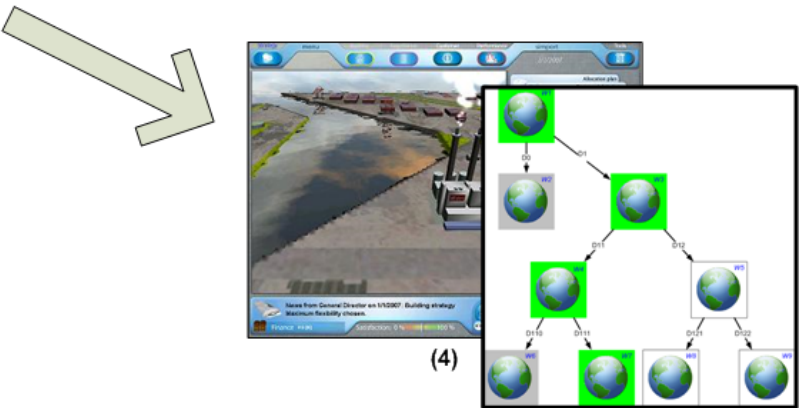

Figure 1: An overview of the discussed scenarios: (1) paper-based approach, (2) studio-based approach, (3) simulation game for spatial decision making, (4) multiple worlds in a simulation game for spatial decision making

\subsection{Multiple worlds in simulations games for trainig}

A group of professionals (e.g. engineers and financial advisors) are in charge of exploring different possibilities on the extension of an existing harbor area. As the case they work on, turns up to be rather unique (they therefore cannot use existing projects as reference material) and tedious, they decide to use a simulation game in order to gain better insight into this complex problem. During the game, a simulation is in charge of displaying what could happen after certain decisions. As the game offers a safe environment to work in, the professionals feel more comfortable to explore more risky decisions. The game offers the possibility to explore these decisions by constructing a tree of possible solutions (depicted in (4) in figure 1). This tree can be branched and pruned and each solution has a different simulation running behind it, which makes it possible to run the different simulations next to each other in order to compare the solutions. This increase of flexibility makes it possible to explore the different possibilities in a single gaming sessions, which helps getting more insight into the problem.

\section{ARCHITECTURE}

An architecture for the proposed simulation game is presented in this section. This architecture reflects our view on how the system of spatial decision making is structured. Based on this view we can subdivide the architecture in 5 components that have to be addressed in a technical implementation: multi-actor environment, game, multiple worlds, visualization, and simulation. An overview of the architecture and its components is depicted in Figure 2.

\subsection{Multi-actor environment}

Various actors (e.g. decision makers, experts, interest group) are involved in a spatial decision making process. The divergence of opinions and interests between those actors is not self-evident yet they have to acknowledge this during the process to successfully cooperate. The ability to cooperate should be supported by the technical implementation. As such we need to look for a virtual environments where the different actors can meet and work together. Different solutions for this have been worked out in current games. The players can be in the same physical room when playing, they can use social software to exchange information during 


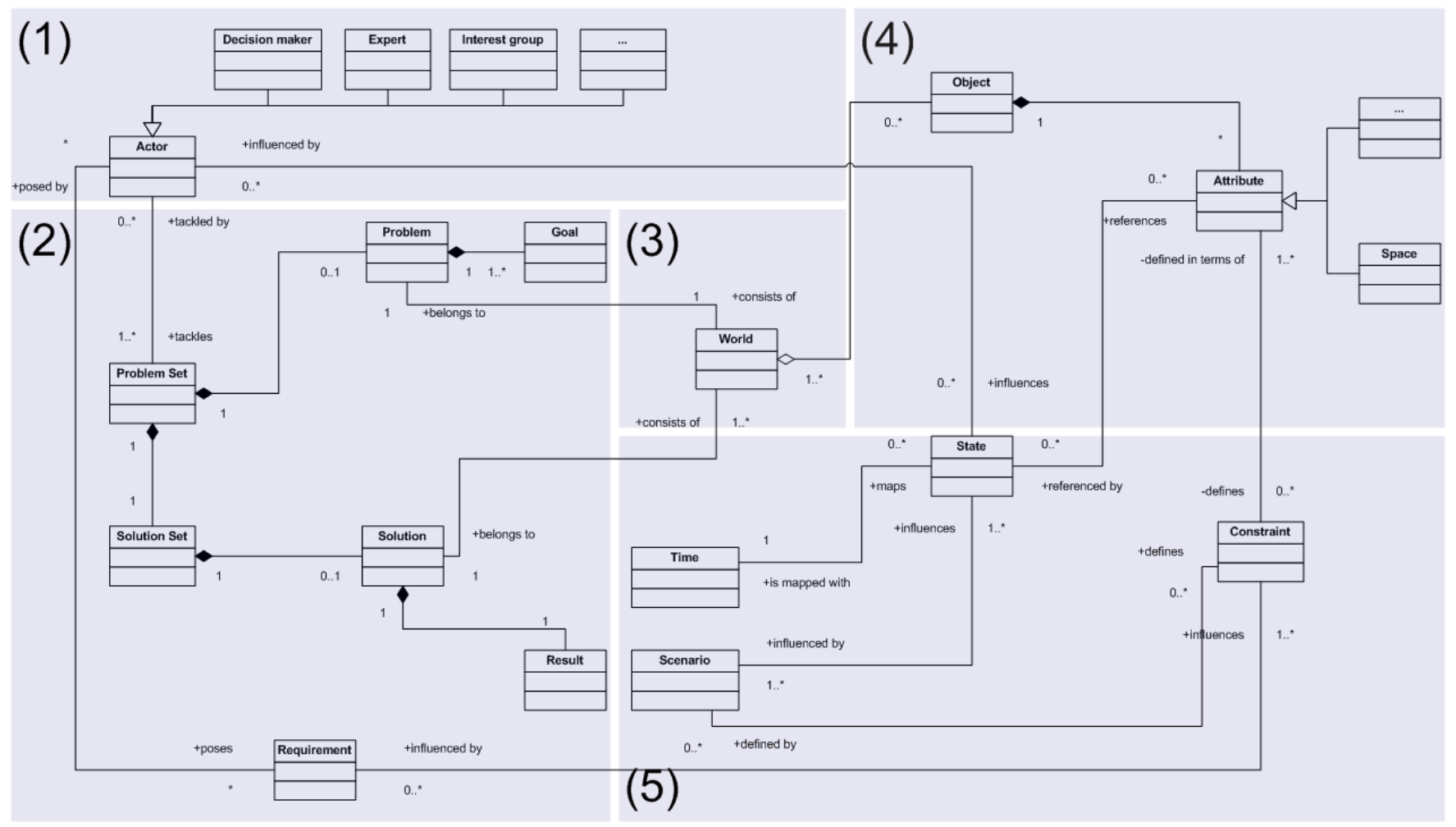

Figure 2: An overview of the architecture with its different components: (1) multi-actor environment, (2) game, (3) multiple worlds, (4) visualization, and (5) simulation.

playing of the game when they are not in the same room, or they can be fully embedded in the game, as we see in virtual worlds such as Second Life (Halloran, Rogers, and Fitzpatrick 2003).

\subsection{Game}

A game is composed of a problem statement out of a problem set with a specific goal for which a solution out of a solution set has to be found existing out of a specific result. As discussed earlier, this result has to be robust. Robustness is assessed by the goals and scenarios. The goals represent conditions that have to be met (e.g. market share, profits, and growth). The scenarios represent exogenous variables (e.g. weather, business competition) that have a strong influence on the conditions in which one has to operate (e.g. a humid climate can ruin machinery, fierce competition can cause bankruptcy). Furthermore, the actors induce specific requirements that have to be met in order to continue with the decision making process. The requirements are translated into constraints used in the simulation. The challenges provided by the goals and scenarios constitute our game.

\subsection{Multiple worlds}

As discussed in section 3 multiple worlds provide a way to assess different decisions. By creating these multiple worlds, different solutions are generated with their corresponding results. The final assessment can then be done by comparing these results.

\subsection{Visualization}

The decision making processes we are focused on have a strong spatial component. As such decisions have to be made with a clear understanding of the space taken by each physical object (e.g. a container, a building) and other attributes belonging to these objects. This is commonly done based on 2D representations of the area of interest, i.e. maps. In many situations, however, 2D representations do not suffice as the height plays an important role. As suggested by Rohrer (2000), visualization is important to understand a complex system. Akpan and Brooks (2005a) and Akpan and Brooks (2005b) extend this further by arguing the need for $3 \mathrm{D}$ visualization to enhance the understanding of the system and to easily spot errors in the model of this system. We therefore argue a $3 \mathrm{D}$ environment is best suitable for our simulation game. 


\section{Fumarola and Verbraeck}

\subsection{Simulation}

A discrete event simulator (Zeigler, Kim, and Praehofer 2000) handles the main flow of time of the game and calculates the future state given the current situation and trends (Nance 1981). States are generated by the flow of time and the events contained in the scenario. The scenario also influences the constraints on the objects. The actual number of simulations, i.e. the instances of a running simulation, will vary as each world, generated by the multiple world mechanism, requires its own simulations. From a software engineering point of view attention will be given to avoid redundant data and simulation runs belonging to the various worlds (e.g. using an architecture as described in Hybinette and Fujimoto (1997)).

\subsection{Summary and considerations}

To construct the proposed simulation game we need a viable architecture that encompasses 5 distinct parts: a multi-actor environment, a game, the multiple worlds concept, visualization and simulation. We showed the interconnections between these parts and how they can function as a whole. To achieve a well functioning implementation special attention has to be given to avoid redundant data and calculations.

\section{FUTURE WORK}

In this paper we described an architecture to design a simulation game to support the training process for spatial decision making in a new and unique way. This architecture has five required parts: a multi-actor environment, a gaming component, the multiple worlds concept, visualization, and simulation. In this paper, we restricted ourselves to the description of the concept of the simulation gaming environment. Future work will be focused on implementing and using this architecture to design simulation games for training spatial decision makers. The actual implementation of the presented concept still remains a challenge due to the large amount of data to be processed and the expected large number of simulation state instances.

\section{REFERENCES}

Akpan, J., and R. Brooks. 2005a. Experimental investigation of the impacts of virtual reality on discrete-event simulation. In Proceedings of the 2005 Winter Simulation Conference, ed. M. Kuhl, N. Steiger, F. Armstrong, and J. A. Joines, 1968-1975: IEEE Computer Society.

Akpan, J., and R. Brooks. 2005b. Practitioners' perception of the impacts of virtual reality on discrete-event simulation. In Proceedings of the 2005 Winter Simulation Conference, ed. M. Kuhl, N. Steiger, F. Armstrong, and J. A. Joines, 1976-1984: IEEE Computer Society.
Bekebrede, G., and I. Mayer. 2006. Build your seaport in a game and learn about complex systems. Journal of Design Research 5 (2): 273-298.

Bouras, C., E. Giannaka, A. Panagopoulos, and T. Tsiatsos. 2006, March. A platform for virtual collaboration spaces and educational communities: the case of eve. Multimedia Systems 11 (3): 290-303.

Chin, R. 2007. Mainport planning suite: Software services to support mainport planning. $\mathrm{Ph}$. D. thesis, Technische Universiteit Delft, Delft, The Netherlands.

Flyvbjerg, B., M. Holm, and S. Buhl. 2004. What causes cost overrun in transport infrastructure projects? Transport reviews 24 (1): 3-18.

Franks, S., F. Gomes, B. Unger, and J. Cleary. 1997. State saving for interactive optimistic simulation. ACM SIGSIM Simulation Digest 27 (1): 72-79.

Galvao, J., P. Martins, and M. Gomes. 2000. Modeling reality with simulation games for a cooperative learning. In Proceedings of the 2000 Winter Simulation Conference, ed. J. Joines, R. Barton, K. Kang, and P. Fishwick, Volume 2, 1692-1698. Orlando, FL, USA: IEEE Computer Society.

Goldsman, D., and B. Nelson. 1998. Comparing systems via simulation. In Handbook of Simulation, ed. J. Banks, Chapter 8, 273-306. New York, NY, USA: John Wiley $\&$ Sons Inc.

Guy, B., N. Bidwell, and P. Musumeci. 2005. GamePlan: Serious gaming for place making. In IE2005: Proceedings of the second Australasian conference on Interactive entertainment, 252: Creativity \& Cognition Studios Press.

Halloran, J., Y. Rogers, and G. Fitzpatrick. 2003. From text to talk: multiplayer games and voiceover IP. In Proceedings of Level Up: 1st International Digital Games Research Conference, 130-142.

Hybinette, M. 2004. Just-in-time cloning. In PADS '04: Proceedings of the eighteenth workshop on Parallel and distributed simulation, 45-51. New York, NY, USA: ACM.

Hybinette, M., and R. Fujimoto. 1997. Cloning: a novel method for interactive parallel simulation. In Proceedings of the 1997 Winter Simulation Conference, ed. S. Andradòttir, K. Healy, D. Withers, and B. Nelson, 444-451. Washington, DC, USA: IEEE Computer Society.

Hybinette, M., and R. Fujimoto. 2001. Cloning parallel simulations. ACM Transactions on Modeling and Computer Simulation 11 (4): 378-407.

Nance, R. 1981. Time and state relationships in simulation modeling. Communications of the ACM 24 (4): 173179.

Pekkola, S. 2002. Critical approach to $3 \mathrm{~d}$ virtual realities for group work. In NordiCHI '02: Proceedings of the 
second Nordic conference on Human-computer interaction, 129-138. New York, NY, USA: ACM.

Rohrer, M. 2000. Seeing is believing: the importance of visualization in manufacturing simulation. In Proceedings of the 2000 Winter Simulation Conference, ed. J. Joines, R. Barton, K. Kang, and P. Fishwick. Orlando, FL, USA: IEEE Computer Society.

Szyliowicz, J., and A. Goetz. 1995. Getting realistic about megaproject planning: the case of the new Denver International Airport. Policy Sciences 28 (4): 347-367.

Vaidman, L. 1998. On schizophrenic experiences of the neutron or why we should believe in the many-worlds interpretation of quantum theory. International Studies in the Philosophy of Science 12:245-261.

Zeigler, B., T. Kim, and H. Praehofer. 2000. Theory of modelling and simulation. 2nd ed. San Diego, CA, USA: Academic Press.

\section{AUTHOR BIOGRAPHIES}

MICHELE FUMAROLA is a Ph.D. student at the Systems Engineering Group of Delft University of Technology. His $\mathrm{Ph}$.D. research is focused on developing simulation games for decision making with a strong visual component. His email address is $<$ m. fumarola@tudelft.nl $>$.

ALEXANDER VERBRAECK is a full professor in the Systems Engineering Group of the Faculty of Technology, Policy and Management of Delft University of Technology, and a part-time full professor in supply chain management at the R.H. Smith School of Business of the University of Maryland. He is a specialist in discrete event simulation for real-time control of complex transportation systems and for modeling business systems. His current research focus is on development of generic libraries of object oriented simulation building blocks in $\mathrm{C}++$ and Java. His e-mail address is $<$ a. verbraeck@tbm.tudelft.nl>, and his web page is <www.tbm.tudelft.nl/webstaf/alexandv>. 